\title{
Exploration of the Electric Field in Clouds
}

$\mathrm{T}^{\mathrm{H}}$ HAT there is electricity in the clouds has been realized since 1755 , when sparks were drawn from the insulated rod set up by D'Alibard and from Franklin's kite, but the problem how to determine with certainty where the electrification was situated had been left unsolved until a method was suggested by Sir George Simpson three years ago and developed at Kew Observatory by $\mathrm{Mr}$. F. J. Scrase.

There has, it is true, been much speculation on the subject, speculation depending on the records of potential near the ground and especially on measurements of the change of gradient produced by lightning flashes. The problem, given the distribution of electricity, deduce the strength of the electric force at a certain point, is easily solved; but the converse problem, given the electric force at a single point; find the distribution, is not soluble. Even if we knew the electric force at every point on the ground, we could not determine the distribution of electricity in the clouds and in the air below the clouds. That is why the interpretation placed upon his records by an investigator has not necessarily been acceptable to his colleagues, and there has been much room for controversy. To gain more intimate knowledge of the state of things in the clouds it was necessary to send up into the clouds apparatus suitable for the purpose. I do not think that this was fully appreciated by anyone until we had tried at Kew Observatory to learn the meaning of the potential gradient records obtained in disturbed weather and had to admit that we were baffled by the ambiguities.

Electrometers had already been sent up in sounding balloons. In 1926 and 1927, Idrac measured the potential gradient in fine weather by means of a valve electrometer and a milliammeter, the indications of which were photographed, but none of his soundings was made through big clouds, and after his untimely death the investigation, which had been carried out at Trappes, was discontinued. For the work at Kew it was anticipated that numerous instruments would be required and they had to be as simple as possible. Sir George Simpson pointed out that it was more important to find the sign of the potential gradient than to measure it, and suggested the use of polefinding paper. That idea was speedily developed, and the first alti-electrograph was sent up a few weeks later. This rapid advance was possible because Mr. L. H. G. Dines had already designed for Sir George Simpson a meteorograph which was intended to detect the presence of vigorous vertical air currents in the clouds. It was only necessary to put on the same spindle as the disk on which the pressure was recorded another disk carrying a piece of prepared paper. During the next two years, some seventy alti-electrographs were sent up from the Observatory.

During an ascent, the electrograph is in series with a pendant wire about twenty metres long, from the end of which there is point discharge whenever the electric field is strong. Two pinpoints make contact with paper impregnated with a solution containing potassium ferrocyanide, and a blue trace is made by one pin or the other as the potential gradient is positive or negative. It was soon found that the thickness of the trace depended on the strength of the gradient, so the records are to a certain extent quantitative as well as qualitative. Most of the ascents were made during thunderstorms or thundery showers, the voltameter not being sensitive to the potential gradients in the clouds which yield moderate rain. To diminish the risk of the alti-electrograph being carried out to sea, a Baker release was used so that the apparatus would be carried down by a parachute from a height of a few kilometres.

The records have been analysed by Sir George Simpson and Mr. Scrase, and their conclusions are set out in a paper recently published by the Royal Society (Proc. Roy. Soc., A, 161, 309-352, August 1937). Some of the balloons sent up were not recovered, and in many cases the electric field was not strong enough to be recorded; but for the period up to the end of 1936 there were thirty-one records available for discussion. Most of the soundings had reached heights of about $7 \mathrm{~km}$. as was intended, but in two cases the balloons had ascended to much greater heights, $15 \mathrm{~km}$. and $17 \mathrm{~km}$., and recorded strong gradients in the stratosphere.

At first glance there is much diversity in the records, but the analysis has yielded a number of valuable generalizations. The first is that in practically every ascent there is a change in the sign of the potential gradient. This is an indication that the balloon passed from below to above a region of concentrated charge. The second generalization is that the location of the charges is related to the temperature of the cloud. These two conclusions are illustrated in a very instructive diagram (Fig. 1).

In this diagram the ascents were arranged in order according to the height at which the 


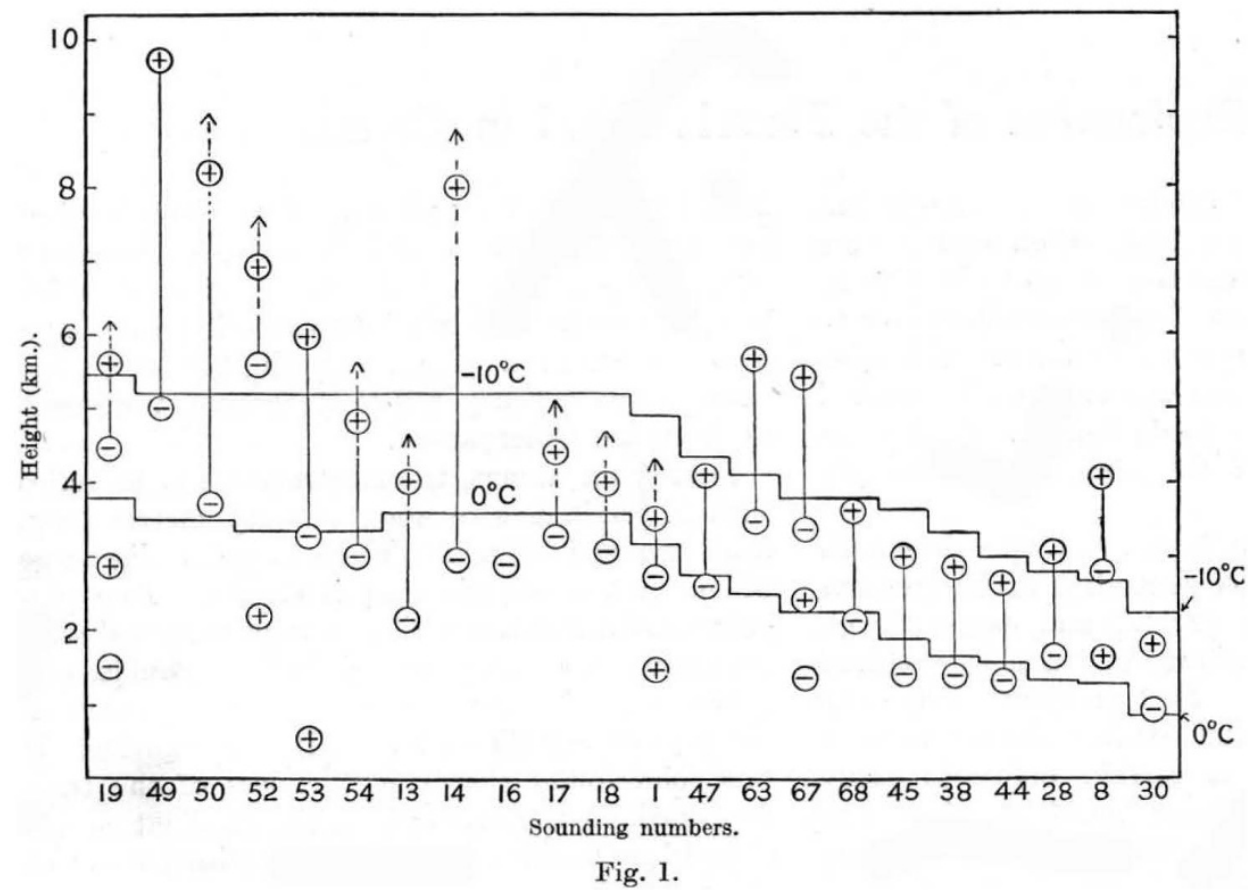

Height of centres of rain mlectrical charges. From Proc. Roy. Soc.,

temperature (calculated by assigning reasonable values to the lapse-rate below and in the cloud) was $-10^{\circ} \mathrm{C}$.

It will be seen that in every case there was evidence for a positive charge near the greatest height reached by the sounding, and, moreover, this positive charge was always in a region where the temperature was below the freezing point. Clearly, positive electrification is developed in the part of the cloud where there is snow and possibly hail. The complementary negative charge is generally near the bottom of the cloud. There are, however, a few cases in which there is a concentration of positive electricity at a low level, an indication that there is a second process at work in the part of the cloud where the snow has melted into raindrops.

Hitherto, in most discussions of the possible ways of generating electricity in the clouds, attention has been concentrated on the behaviour of raindrops, in spite of the fact that the thunder-cloud is usually capped by false cirrus and in spite of the general A, 161, 309-352 (1937).

acceptance in recent $y e a r s$ of $t h e$ doctrine, strongly advocated by Bergeron, that rain is nearly always melted snow. The discovery that electrification is produced in the region in which there is snow recalls, however, the early theory of thunderstorms developed by L. Sohncke, of Munich, in a pamphlet published at Jena in 1885 (cf. Met. Z., $5,413-425 ; 1888)$. Sohncke maintained that the electrification was produced by friction between ice and water, the ice acquiring a positive charge, the water a negative charge. If this theory had been correct, the negative charge on the cloud particles would have been carried upwards by convection currents and the snow would have carried a positive charge downwards. Actually the positive charge is found at the top of the cloud, so Sohncke's theory cannot be valid.

Simpson and Scrase remark that when there is driving snow near the ground, a strong positive gradient is developed. This was demonstrated by Simpson in the Antarctic and confirmed by Sverdrup during the voyage of the Maud in the Arctic

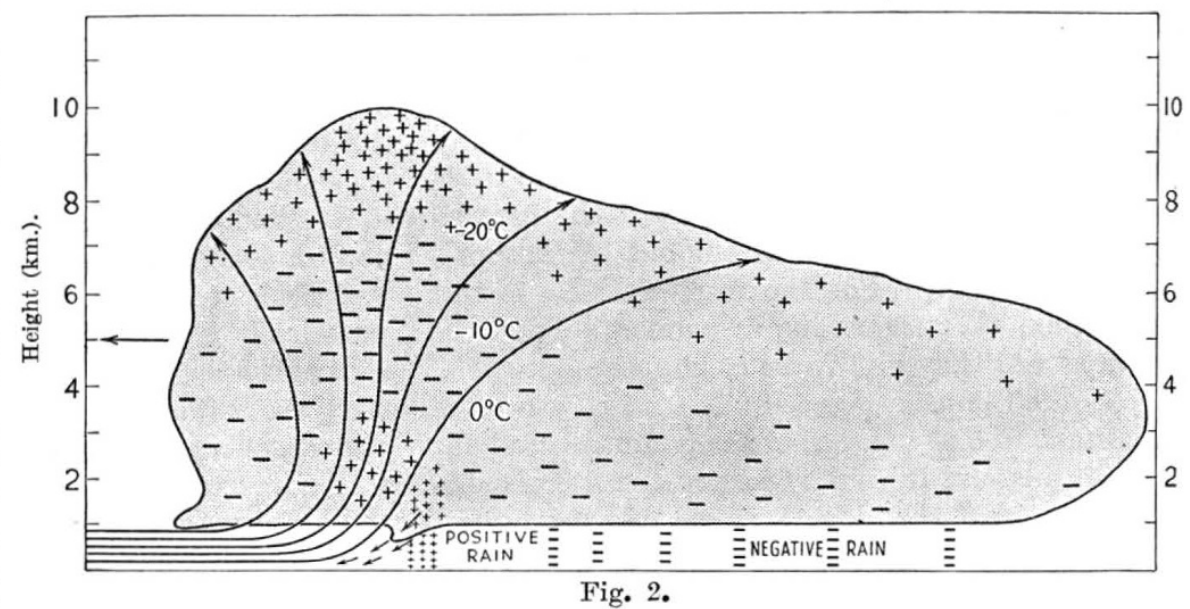

Generalized diagam SHOWING AIR CURRENTS AND Distribution of ELECTRICITy IN A TYPicAl heAT Thunderstorm. From Proc. Roy. Soc. A, 161, 309-352 (1937). 
Ocean. It is likely that when snow particles collide, the particles acquire a negative charge, whilst a positive charge is given to the air. The snow falls to the ground and a positive space charge remains in the air and may extend to a considerable height. The process is analogous to what happens in a sandstorm, though the sign of the electrification is reversed. Thus it is to be expected that in the part of a cloud where there are snow particles in violent motion there will be electrification, that the snow will carry down a negative charge and that the air streaming upwards will take a positive charge to the top of the cloud, this charge being communicated rapidly to the cloud particles. It may be added that as the cloud-top is in a region where the conductivity is high, a strong current of positive electricity can flow upwards to the Heaviside layer, so providing (in accordance with Wilson's theory) for the air-earth current of fine weather.

There is some evidence that the regions in which positive electrification is produced low down in the clouds are those where the rain is heaviest, and Simpson and Scrase hold that the breaking-drop theory of the former is the explanation of this electrification. As the snow melts, it forms large drops, and if these are too large for stability they will break up. The rising current of air will carry a negative charge and the positively charged droplets fall or perhaps remain nearly stationary. The hypothesis that this process occurs only where the upward current is strong is embodied in Fig. 2.

Perhaps the most surprising result of the investigation is that no evidence is found for very strong fields in the clouds. The magnitude of the potential gradient is determined by a tentative formula which takes account of the width of the trace on the electrogram and also of the density of the air. It was found that when the gradient exceeded about 100 volts per centimetre, the trace was marked by sparking, but this occurred in only a few of the ascents. In 1925 Wilson put forward the hypothesis that, when a lightning flash is about to occur, the electric field just below a thundercloud is probably nearly 10,000 volts per centimetre, a hundred times the strength near the ground. The hypothesis seemed to be supported by estimates of the space charge produced by point discharge from trees and other objects. The soundings indicate, however, that generally there is little if any increase in the gradient as the cloud is approached. It may be that the estimates are systematically in error, and it is to be hoped that control measurements will be made by some more direct method.

One reason for suspecting such systematic error is that the estimated gradients imply that the charges in the clouds are comparatively small. If the gradients over two planes a kilometre apart are $+100 \mathrm{v} . / \mathrm{cm}$. and $-100 \mathrm{v} . / \mathrm{cm}$. respectively the charge is (by Coulomb's law) only 0.2 coulombs per cubic kilometre. This is insignificant compared with 20 coulombs, the quantity of electricity supposed to be discharged by a lightning flash. In what part of a cloud the lightning originates, and why it occurs, we have yet to discover.

The investigation is being continued at Kew and, although the ascents made during 1937 have not led to any striking new discoveries, it may be that in the course of time a record will be obtained which will provide the missing clue.

F. J. W. WHIPPLE.

\title{
Heat Production, Nutrition, and Growth in Man: Some New Views*
}

\author{
By Dr. E. P. Poulton
}

\section{INDIRECT AND DiRECT CALORIMETRY}

$\mathrm{I}^{\mathrm{N}}$ $\mathrm{N}$ estimating the heat production of an individual, the oxygen intake and the carbon dioxide output are measured. After allowing for protein metabolism the oxygen intake is converted into calories of heat generated, by multiplying it with a factor which depends on the respiratory quotient (volume of carbon dioxide produced/volume of oxygen absorbed). These multiplying factors were

- Substance of the presidential address delivered to Section I (Physiology) of the British Association at Nottingham on September 2, revised and modifled. calculated originally by Zuntz and Schumburg (1901), and are based upon: (a) the heats of combustion of carbohydrate and fat; $(b)$ the chemical composition of carbohydrate and fat; and $(c)$ the theory that when the respiratory quotient has the value unity carbohydrate alone is oxidized in the body and when the respiratory quotient has a value equal to 0.707 fat only is being oxidized, and at intermediate values of the respiratory quotient corresponding proportions of carbohydrate and fat are being oxidized. This method of calculating the heat produced by an individual is known as 